\title{
Mitochondrial dysfunction in alveolar and white matter developmental failure in premature infants
}

Vadim S. Ten ${ }^{1}$

At birth, some organs in premature infants are not developed enough to meet challenges of the extra-uterine life. Although growth and maturation continues after premature birth, postnatal organ development may become sluggish or even arrested, leading to organ dysfunction. There is no clear mechanistic concept of this postnatal organ developmental failure in premature neonates. This review introduces a conceptforming hypothesis: Mitochondrial bioenergetic dysfunction is a fundamental mechanism of organs maturation failure in premature infants. Data collected in support of this hypothesis are relevant to two major diseases of prematurity: white matter injury and broncho-pulmonary dysplasia. In these diseases, totally different clinical manifestations are defined by the same biological process, developmental failure of the main functional units - alveoli in the lungs and axonal myelination in the brain. Although molecular pathways regulating alveolar and white matter maturation differ, proper bioenergetic support of growth and maturation remains critical biological requirement for any actively developing organ. Literature analysis suggests that successful postnatal pulmonary and white matter development highly depends on mitochondrial function which can be inhibited by sublethal postnatal stress. In premature infants, sublethal stress results mostly in organ maturation failure without excessive cellular demise.

W hite matter injury (WMI) and broncho-pulmonary dysplasia (BPD) are the most devastating diseases of prematurity, accounting for the majority of all life-long disabilities associated with premature birth. Neuroimaging studies detect white matter abnormalities in $~ 50 \%$ of infants and children born with the birth weight less than $1,500 \mathrm{~g}$ (very low birth weight (VLBW)). These WM abnormalities account for neurological deficits observed in the approximately $90 \%$ of the affected infants (1). In the United States, BPD annually impacts health of $\sim 10,000$ premature infants. These infants exhibit lifelong alteration of their pulmonary function (2). Thus, WMI and BPD are responsible for enormous socio-economic burden.

WMI consists of a spectrum of cerebral abnormalities in which defect of axonal myelination is the most common pathological finding. Clinically, WMI manifests with various degrees of permanent neurological deficit. In BPD, primary pathological lesion is poor pulmonary alveolarization secondary to developmental arrest of alveolar formation. This disease manifests with pulmonary insufficiency in maintaining adequate gas exchange. Because of our incomplete understanding of the mechanisms of these diseases, the progress in treatment and prevention of BPD and WMI remains stagnant. What is known, that both these diseases are unique to premature infants and that organ developmental failure is a key pathophysiological mechanism. This knowledge may be interpreted as a cue for existence of a common cellular mechanism in the evolution of BPD and WMI.

\section{THE EVOLUTION OF WMI AND BPD IS SIMILAR}

Epidemiological data suggest that cystic periventricular leukomalacia (PVL) is no longer a predominant form of the WMI and occurs only in $\sim 5 \%$ of VLBW infants. In contrast, diffuse or focal noncystic WMI affects $\sim 50 \%$ of VLBW infants $(1,3)$. Clinical presentation of WMI has changed accordingly. Premature infants, now survive with milder spectrum of sensorimotor and cognitive disabilities (4). These data imply that pathological pattern of WMI has shifted away from acute and massive degeneration of neurons, oligodendrocytes (OLs) and other cells, known as cystic PVL, toward diffuse or focal noncystic WMI. The latter form of WMI is mostly characterized by axonal hypomyelination, without extensive cerebral tissue loss. It is possible, that progress in medical care of premature infants contributed to this shift in neuropathological and clinical presentation of WMI. While cystic PVL is defined by acute loss of the white matter parenchyma following a lethal insult, noncystic WMI is associated with maturational failure of OLs precursors to reach their myelin-producing state $(3,5)$. To date, clinical causes of cystic PVL: acute and lethal cerebral insults, hypoxia-ischemia, intraventricular/parenchymal hemorrhage as well as primary molecular mechanisms of cellular death have been extensively studied and largely defined. In contrast, clinical causes and molecular mechanisms for maturational arrest of surviving OLs received much less attention and remain unclear.

Interestingly, natural evolution in pathological and clinical presentation of neonatal BPD is strikingly similar to that of 


\section{Mitochondria and diseases of prematurity}

WMI. Not long ago, neonatologists also recognized a shift in the pathological and clinical patterns of the same disease. This shift was defined as the evolution from the "Old" BPD to the "New" BPD $(6,7)$. Similarly to the difference between cystic PVL and noncystic WMI, the difference between these two patterns of BPD consists of changes in pathological and clinical presentation. The pathological hallmark of "Old" BPD was described as a massive cellular demise (necrosis/apoptosis) followed by inflammatory response and extensive fibrosis of the lung parenchyma (8). This pathological phenotype of "Old" $\mathrm{BPD}$ is very similar to that of cystic PVL; formation of periventricular cystic lesions in response to a massive loss of the WM parenchyma. Primary pathological presentation in "New" BPD is mostly limited to poor development of alveoli which functionally, resembles poor axonal myelination in noncystic WMI. Thus, BPD and WMI exhibit similar evolution of their classical pathological manifestation, by shifting away from the extensive loss of parenchymal tissue toward developmental arrest of the organ's main functional units. In both diseases, these changes were recognized at the same "developmental" stage of current neonatology. This suggests that BPD and WMI may share common subcellular mechanism, contributing to developmental failure of lungs and brain, and this mechanism is sensitive to a progress in neonatal medical care.

\section{MITOCHONDRIAL DYSFUNCTION AND MATURATION FAILURE OF LUNGS AND WHITE MATTER}

In humans and other mammals, neonatal age is the time of the highest energy requirement. One of the main purposes of cellular bioenergetics in this age is generation of energy for rapid organs growth and development. At birth, lungs of the VLBW premature infants are at the saccular stage of their maturation and not fully functional. Similarly, brains of premature neonates are structurally and functionally immature. This is evidenced by high incidence of central apnea, poor axonal myelination, and by the presence of mostly immature oligodendrocytes (OLs) (9). Even at term gestation, human brain contains only $50 \%$ of mature myelin-producing OLs $(3,10)$. The developmental spurt requires significant amount of energy. It is not surprising that in rats, during the initial $3 \mathrm{wk}$ of life, when the rate of cerebral growth is the greatest relatively to any other age (11), total mitochondrial capacity in the brain increases by 11-fold, compared to that at birth (12). At cellular level, differentiation and maturation requires up to $45 \%$ increase in adenosine-triphosphate (ATP) consumption rate (13). Differentiating OLs metabolically are extremely active, significantly contributing to an increase in total cerebral metabolic demand during brain development (14). In regards to the white matter maturation, it has been calculated, that the energy cost of myelination in rodent optic nerve is equal to the energy spent for generation of 15-23 million action potentials in myelinated optic nerve (15).

Similarly to the brain, pulmonary development and growth also requires substantial energy support. For example, leptindeficient mice, known for their low metabolic rate, demonstrated significantly poorer alveolarization and lung volume

\section{Integrated Mechanism Review}

at 2 wk of life (16). Mitochondrial dysfunction, defined by an inhibition of oxidative phosphorylation, has been implicated in the mechanisms of poor proliferation of alveolar epithelium (17). The importance of intact mitochondrial bioenergetics function for proper alveolarization can be supported by our recent studies. In the hyperoxia-induced or mechanical ventilation-induced models of BPD-like lung injuries, alveolar developmental arrest was strongly associated with depression of the respiratory chain in pulmonary mitochondria $(18,19)$. Moreover, pharmacological inhibition of mitochondrial complex-I or partial uncoupling of lung mitochondria, without any pulmonary stress (hyperoxia or mechanical ventilation), fully replicated the phenotype of "new BPD", an arrested alveolarization $(18,19)$. These preclinical studies suggest that inhibition of mitochondrial bioenergetics in the developing lungs may represent a fundamental mechanism of alveolar developmental arrest in infants with BPD.

In regards to the role of mitochondrial dysfunction in the failure of white matter maturation, Bizzozero et al., using various inhibitors of mitochondrial respiration in young rats have shown that formation and compaction of the myelin sheath was highly ATP-dependent (20). In adult rats, however, the maintenance of myelin sheath was nearly ATP-independent (20). Interestingly, while undifferentiating Oligodendrocyte precursor cell (OPC) exhibited resistance to inhibition of mitochondrial complex-I, actively differentiating OPC have arrested their maturation in response to even very mild, sublethal inhibition of mitochondrial complex-I (21). In contrast, protection of complex-I with hyperforine preserved mitochondrial ADPphosphorylating activity and increased expression of the OLs maturation-related proteins (22). These data indicate that normal differentiation of OPC is very sensitive to sublethal mitochondrial dysfunction. An inhibition of complex-IV which resulted in lethal mitochondrial dysfunction not only arrested OLs differentiation but, as expected, caused degeneration of mature OLs and their precursors (23). Taken together, above referenced reports suggest that lethal or sublethal mitochondrial dysfunctions negatively affect OLs maturation, regardless of the extent of OLs demise. It is possible that severity of mitochondrial dysfunction, lethal or sublethal, defines pathological phenotype of WMI: cystic PVL or diffuse hypomyelination. The idea to connect cellular energy state with pathogenesis of WMI is not novel. Earlier, Rosenberg et al. proposed potential role of neonatal undernutrition in diffuse cerebral hypomyelination in WMI (24). Importantly, there is a specific time of white matter vulnerability to caloric restriction in undernourished subjects (25). This specific time coincides with the age of the most active axonal myelination (24). While potential mechanisms for the starvation-induced axonal hypomyelination could be a deficit of precursors to maintain robust myelin synthesis (24), undernutrition also inhibits OLs maturation, and thereby myelination (26). Nutrition is not only a source of substrates for structural (myelin) synthesis. It is also criti$\mathrm{cal}$ for generation of energy. It is not unreasonable to propose, that poor axonal myelination in undernourished subjects was caused by suboptimal energy support of OPC maturation and 


\section{Integrated Mechanism Review}

myelin formation. In well-nourished subjects, however, mitochondrial dysfunction can lead to bioenergetic deficit for a proper organ development. Our preliminary data show that in the same neonatal mouse, alveolar developmental arrest and diffuse axonal hypomyelination can be achieved by exposure to mitochondrial uncoupling agent, 2'4' dinitrophenol (Figure 1). This fact that isolated mitochondrial stress fully reproduced human phenotype of diffuse WMI and "new" BPD in the same animals (Figure 1) supports the hypothesis, that mitochondrial bioenergetics dysfunction is a common subcellular mechanism of postnatal developmental failure of these organs.

Of interest, inherited defects of mitochondrial function affect multiple organs development. Although, clinical presentation of these diseases differs, depending upon the site and the severity of mitochondrial dysfunction, signs of organs developmental failure always exist in these patients. Neuronal migration defect, lack of axonal projection, and axonal hypomyelination are the most common cerebral presentations of congenital mitochondrial diseases (27). White matter abnormalities and cortical dysplasia are now increasingly recognized as manifestations of inborn mitochondrial defects (27). Patients with mutations in complex-I have a 100 -fold increased risk of severe demyelination secondary to multiple sclerosis (28). In lungs, pulmonary hypertension and right ventricular cardiomyopathy due to under-development of alveolar vasculature is one of the lethal presentations of congenital cytochrome $\mathrm{C}$ oxidase deficiency (29). Respiratory distress is a classical presentation of Stuve-Weidemann syndrome associated with partial deficiency of complex-I and complex-IV activities (30).

\section{POSTNATAL CAUSES OF MITOCHONDRIAL DYSFUNCTION IN THE DEVELOPING LUNGS AND BRAIN}

This review is focused only on sublethal mitochondrial dysfunction as a potential mechanism for the brain and lungs

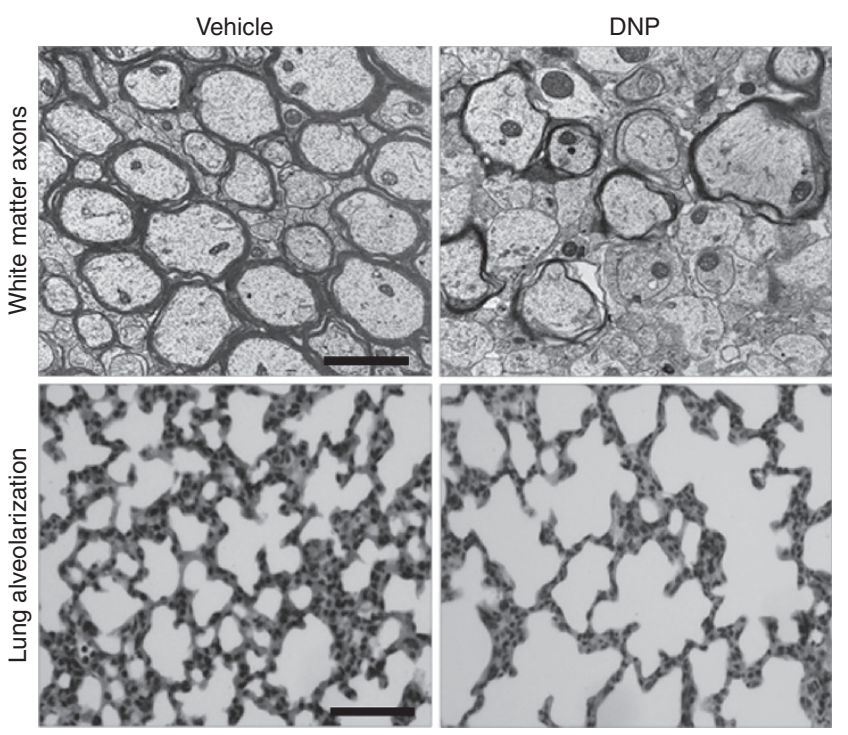

Figure 1. Electron microscopy of axons in the corpus callosum and $\mathrm{H}$ and $E$ staining of lungs in the same mice treated with either vehicle or $2^{\prime} 4^{\prime}$ dinitrophenol ( $2 \mathrm{mcg} / \mathrm{g}$ ). Scale bar: $1 \mu \mathrm{m}$ (brain) and $50 \mu \mathrm{m}$ (lungs).

\section{Ten}

maturation failure. Therefore, extensively reviewed mitochondrial dysfunction-driven mechanisms of cellular death following lethal insult, ischemia-reperfusion injury $(31,32)$, and other brain or lung injuries with massive parenchymal tissue loss are not discussed.

Since immature lungs are dysfunctional, VLBW infants always require respiratory support. The most common mode of respiratory support is supplemental oxygen therapy, delivered with or without mechanical ventilation. While supplemental oxygen normalizes systemic oxygenation, lungs experience exposure to toxic levels of oxygen. Hyperoxic exposure to the developing lungs has been shown to inhibit pulmonary mitochondrial respiration and ATP-generation rate (18). Similarly, another mode of respiratory support, mechanical ventilation, especially with excessive tidal volume, significantly depressed mitochondrial ADP-phospholylating activity in neonatal mice (19). In vitro, direct shear stress which mimics barotrauma of mechanical ventilation inactivated mitochondrial respiratory chain, and superimposed hyperoxic exposure exacerbated mitochondrial dysfunction in pulmonary endothelial cells. Both these effects were associated with increased production of superoxide and peroxynitrite in mitochondria (33), suggesting self-oxidative mitochondrial injury. In human pulmonary epithelial-like A549 cells, 96h of hyperoxic exposure significantly depleted ATP content, dissipated mitochondrial membrane potential and inhibited mitochondrial respiration (34). All these signs of mitochondrial dysfunction were associated with arrested cellular proliferation and excessive cellular death (35). Some authors, using in vivo or in vitro paradigms of hyperoxic exposure in neonatal mice or in primary culture of pulmonary endothelial cells have reported, that hyperoxia primarily affected complex-I activity in the respiratory chain, causing an inhibition of NAD-linked (complex-I dependent) mitochondrial respiration (19,35). Others, using cultured MLE12 cells exposed to hyperoxia and lung mitochondria isolated from mice exposed to hyperoxia have shown inhibited complex-I and complex-II-dependent respirations with complex-IV activity being intact (36). Although, more studies addressing sensitivity of specific mitochondrial complexes to hyperoxia yet to come, to date, experimental data clearly demonstrate that hyperoxia negatively affects respiratory chain in lung mitochondria.

Mechanistically, oxygen toxicity is viewed as an oxidative stress. Oxygen radicals and products of lipid peroxidation can directly deteriorate mitochondrial function by inhibiting oxidative phosphorylation, by reducing membrane potential and aconitase activity in pulmonary epithelial cells (37). Mitochondria-targeted antioxidant, mitoTEMPO, attenuated the extent of hyperoxia-induced lung injury and right ventricular hypertrophy in neonatal mice (38). This suggests that reactive oxygen species (ROS) originating in mitochondria contribute to the BPD-like injury. Indeed, during chemical toxic stress in vitro, mitochondria-originated ROS are capable of mitochondrial self-oxidation, the event which promotes oxidative injury in the lungs $(39,40)$. Interestingly, in the mitochondria isolated from the brain, hyperoxia also dramatically accelerates mitochondrial ROS production, 


\section{Mitochondria and diseases of prematurity}

causing mitochondrial dysfunction secondary to self-oxidative damage (41). Some authors challenge deleterious role of ROS originating in pulmonary mitochondria during hyperoxic lungs exposure (42), while others reported a greater oxidative response of pulmonary mitochondria to hyperoxia in neonatal versus adult rodents (43). More studies are needed to determine whether mitochondria-originated ROS mediate oxygen toxicity in the developing lungs.

Given that BPD often results in chronic hypercapnia, it is worth to mention that elevated $\mathrm{CO}_{2}$ level inhibited mitochondrial oxidative phosphorylation and impaired proliferation of pulmonary epithelial cells in culture (17). The latter was rescued by $\alpha$-ketoglutarate or overexpression of mitochondrial isoform of isocitrate dehydrogenase (17), suggesting that hypercapnia inhibits enzymes of the Kreb's cycle. Thus, hyperoxic exposure during oxygen therapy and shear stress of mechanical ventilation, as well as hypercapnia can be considered as clinical causes of pulmonary mitochondrial dysfunction in premature infants.

In regards of potential clinical triggers of nonlethal mitochondrial dysfunction in cerebral white matter, clinical relevance of existing experimental evidence served as selection criteria for discussion. Artificial alteration of cerebral oxygenation, hyperoxemia or hypoxemia, has been extensively used to model WMI in animals. It is important to realize, that oxygen therapy in premature human neonates is used only to reach and maintain normoxemia. Therefore, reported models of chronic hyperoxia-induced WMI in neonatal rodents $(44,45)$ are not considered for discussion. Experimental studies also showed that postnatal exposure to chronic hypoxia $\left(10 \% \mathrm{O}_{2}\right.$ for 8-10 d) reproducibly replicated human phenotype of diffuse WMI (46-48). It has to be noted, however, that in human premature infants the use of the respiratory support maintains systemic and cerebral oxygenation at the physiological level of normoxemia. Therefore, clinical relevance of paradigms using chronic hypoxic exposure as potential cause of WMI in humans is restricted to a very small subset of premature infants in whom respiratory support is ineffective. In addition, compensatory physiological changes triggered by chronic hypoxia were not characterized (49). This questions clinical utility of this widely used postnatal model of WMI (49). In contrast to chronic hypoxemia, another potential clinical cause of diffuse WMI, intermittent hypoxemia (IH), is the most common clinical manifestation of prematurity, especially, if prematurity is complicated by the BPD $(50,51)$. This fact makes $\mathrm{IH}$ paradigm highly relevant to real clinical scenario. There is a line of experimental evidence that IH stress results in diffuse WMI in neonatal rodents (52-54). If the hypothesis proposed in this review is viable, then $\mathrm{IH}$ and chronic hypoxia should negatively affect mitochondrial function in the brain and, specifically, in the OLs lineage cells. Although, acute hypoxic inhibition of cerebral mitochondria has been extensively reported (55-57), it was surprising to find virtually no reports on alteration of mitochondrial function in OLs and their precursors in response to sublethal intermittent or chronic hypoxic stress. What has been shown, that severe intermittent hypoxemia/

\section{Integrated Mechanism Review}

hypercapnia significantly inhibited respiratory chain in the mitochondria isolated from the entire brain, and this was associated with acute subcortical and cortical brain injury (54). Our preliminary data demonstrated, that in neonatal mice, mild (without bradycardia and alteration of the cerebral blood flow), nonlethal intermittent hypoxemic stress significantly uncoupled mitochondrial respiration, not only in the organelles isolated from subcortical regions of the brain, but also in mitochondria of OLs-enriched cerebral cell suspension. Importantly, the same non-lethal intermittent hypoxemic stress fully replicated human noncystic WMI phenotype; diffuse axonal hypomyelination and permanent sensorimotor deficit (53). Of interest, upon exposure to hypoxic stress, mature OLs in culture demonstrated significant reduction in their energy utilization, particularly in glycolitic ATP production. This was associated with cessation of myelin production, while favoring OLs survival (58). Thus, it is possible that nonlethal chronic hypoxemia or IH-stress negatively affect mitochondrial bioenergetic function in mature, differentiating OLs and their precursors which eventuates in poor axonal myelination in premature infants.

Another potential mechanism, altering OLs mitochondria in non-cystic WMI, is neuroinflammation. Usually, the neuroinflammation, that affects WM development, develops in response to acute and cell-fatal cerebral insult. Mitochondrial dysfunction following an acute ischemia or any other lethal insult is well documented and expected $(31,32,59)$. Our focus is mitochondrial dysfunction triggered by the inflammation disseminated to intact immature brain. Developing brain may become a distant organ-target for systemic inflammation during neonatal sepsis, or fetal systemic inflammatory response syndrome, or other extensively inflamed organ (lungs, intestine, bone marrow). For example, one of the key mechanistic roles in BPD was assigned to pulmonary inflammation which, theoretically, is spreadable to the brain. While focal neuroinflammation has been implicated in the pathogenesis of cystic PVL and diffuse WMI (59-62), there are no direct evidence that generalized inflammation, originating in the BPDaffected lungs or other organ, can cause developmental arrest of cerebral myelination or alter OLs mitochondrial function. However, increased microglial activation and astrocytosis, alone with reduced OLs density in subcortical WM following prolonged mechanical ventilation has been reported in the primate model of BPD $(63,64)$. Because systemic oxygenation was not affected in these animals, one may conclude that prolonged mechanical ventilation is associated with WMI via the mechanism different from hypoxemia. It has been shown, that local inflammation induced by the hind-limbs ischemia-reperfusion injury disseminates into the brain, causing significant activation of microglia in neonatal mice (65). It is conceivable that circulating chemokines and cytokines originated in other organs trigger neuroinflammation leading to myelination defect in the developing brain. Mechanistically, inflammatory mediators can promote WMI through vasoactive action that involves hypoxia-ischemia or by direct or indirect toxicity to OLs or their precursors (49). One of the potential 


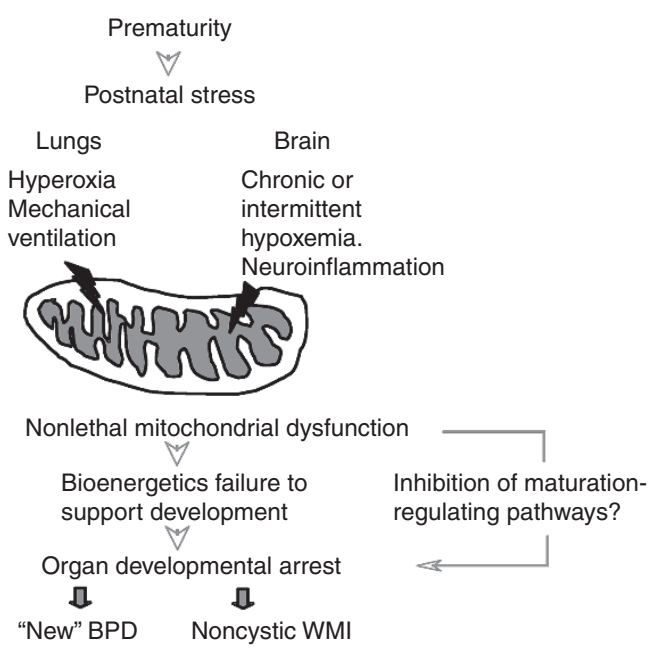

Figure 2. Hypothetical mitochondria-driven mechanisms for postnatal developmental failure of lungs and cerebral white matter in premature infants.

mechanisms for direct toxicity of inflammatory mediators could be an inhibition of mitochondrial bioenergetics in OLs lineage. This hypothesis can be supported by the study where low $(10 \mathrm{ng} / \mathrm{ml})$, nonlethal dose of TNF- $\alpha$ arrested maturation of cultured OPC (NG2-cells) by altering mitochondrial $\mathrm{Ca}^{2+}$ buffering capacity and membrane potential (66). This phenotype of in vitro arrested OPC maturation was also replicated simply by the exposure of NG2 cells to mitochondrial uncoupler (66). Interestingly, above referenced report indirectly argues against deleterious role of mitochondrial ROS in this paradigm, because mitochondrial uncoupling and dissipation of membrane potential dramatically reduces ROS generation capacity of mitochondria (67). While, potential mechanistic role of mitochondrial ROS in the neuroinflammation-driven WMI needs to be determined, an inhibition of oxidative phosphorylation via targeting of subunit-I in cytochrome $\mathrm{c}$ oxidase by TNF- $\alpha$ has been shown (68). Of note, experimental augmentation of mitochondrial function with mitochondrial metabolites or mitochondrial chaperone ( $\mathrm{mtHsp} 75 /$ mortalin) partially preserved in vitro and in vivo neurogenesis impaired by the inflammation (69). Based on presented data, one may speculate that inflammation disseminated from other organs into intact developing brain affects OLs mitochondrial function, leading to myelination defect.

\section{SUMMARY}

Analysis of published reports suggests that exogenous stress to the actively developing lungs $\left(\mathrm{O}_{2}\right.$-toxicity, baro-trauma of mechanical ventilation) or to the immature brain (intermittent or chronic hypoxia, neuroinflammation) may cause mitochondrial dysfunction in these organs. This mitochondrial dysfunction is not lethal. Therefore, the majority of parenchymal cells remain viable. However, their survival may be possible at the expense of arrested cellular differentiation and proliferation, resulting in poor postnatal organs development (Figure 2). This maturational failure may become permanent, if mitochondrial dysfunction coinsides with natural "window" of developmental opportunity. Testing this hypothesis requires clinical and experimental evidence for: (i) postnatal stress-driven mitochondrial bioenergetics dysfunction in cells responsible for pulmonary and white matter maturation, (ii) direct or/and indirect dependence of lungs and white matter development upon mitochondrial bioenergetics. While direct dependence upon mitochondrial ATP-generating capacity will support essential role of bioenergetics in normal growth and development, indirect dependence of organs maturation upon mitochondrial bioenergetics will highlight novel signaling role of mitochondria in organs development. For example, it has been shown that low dose of TNF- $\alpha$, without alteration of mitochondrial ATP production, has arrested rat OPC maturation in culture by altering mitochondrial membrane potential, $\mathrm{ROS}$ production, and $\mathrm{Ca}^{2+}$ oscillations (66). These signs of mitochondrial dysfunction were coupled with inhibition of the mammalian target of rapamycin (mTOR) (70), the pathway reported to be essential for OLs maturation (71). Experimental prevention or attenuation of WMI and BPD manifestation by preservation of mitochondrial function will serve as therapeutic proof of the hypothesis and may emerge as novel therapeutic strategy against diseases of postnatal developmental failure in premature neonates.

\section{STATEMENT OF FINANCIAL SUPPORT}

This study was supported by internal bridge funding award UR009347, Department of Pediatrics, Columbia University and by R01 grant NS 099109.

Disclosure: The author has nothing to disclose.

\section{REFERENCES}

1. Khwaja O, Volpe JJ. Pathogenesis of cerebral white matter injury of prematurity. Arch Dis Child Fetal Neonatal Ed 2008;93:F153-61.

2. McEvoy CT, Jain L, Schmidt B, Abman S, Bancalari E, Aschner JL. Bronchopulmonary dysplasia: NHLBI Workshop on the Primary Prevention of Chronic Lung Diseases. Ann Am Thorac Soc 2014;11 Suppl 3:S146-53.

3. Volpe JJ, Kinney HC, Jensen FE, Rosenberg PA. The developing oligodendrocyte: key cellular target in brain injury in the premature infant. Int J Dev Neurosci 2011;29:423-40.

4. Back SA. Brain injury in the preterm infant: new horizons for pathogenesis and prevention. Pediatr Neurol 2015;53:185-92.

5. Buser JR, Maire J, Riddle A, et al. Arrested preoligodendrocyte maturation contributes to myelination failure in premature infants. Ann Neurol 2012;71:93-109.

6. Ambalavanan N, Carlo WA. Bronchopulmonary dysplasia: new insights. Clin Perinatol 2004;31:613-28.

7. Mosca F, Colnaghi M, Fumagalli M. BPD: old and new problems. J Matern Fetal Neonatal Med 2011;24 Suppl 1:80-2.

8. Coalson JJ. Pathology of bronchopulmonary dysplasia. Semin Perinatol 2006;30:179-84.

9. Ortinau C, Neil J. The neuroanatomy of prematurity: normal brain development and the impact of preterm birth. Clin Anat 2015;28:168-83.

10. Back SA, Luo NL, Borenstein NS, Levine JM, Volpe JJ, Kinney HC. Late oligodendrocyte progenitors coincide with the developmental window of vulnerability for human perinatal white matter injury. J Neurosci 2001;21:1302-12.

11. Dobbing J, Sands J. Comparative aspects of the brain growth spurt. Early Hum Dev 1979;3:79-83.

12. Erecinska M, Cherian S, Silver IA. Energy metabolism in mammalian brain during development. Prog Neurobiol 2004;73:397-445.

13. Kostifa MM, Zivkovifa RV, Rapoport SM. Maturation-dependent changes of the rat reticulocyte energy metabolism and hormonal responsiveness. Biomed Biochim Acta 1990;49:S178-82 


\section{Mitochondria and diseases of prematurity}

14. Amaral AI, Hadera MG, Tavares JM, Kotter MR, Sonnewald U. Characterization of glucose-related metabolic pathways in differentiated rat oligodendrocyte lineage cells. Glia 2016;64:21-34.

15. Harris JJ, Attwell D. The energetics of CNS white matter. J Neurosci 2012;32:356-71.

16. Huang K, Rabold R, Abston E, et al. Effects of leptin deficiency on postnatal lung development in mice. J Appl Physiol (1985) 2008;105:249-59.

17. Vohwinkel CU, Lecuona E, Sun H, et al. Elevated $\mathrm{CO}(2)$ levels cause mitochondrial dysfunction and impair cell proliferation. J Biol Chem 2011;286:37067-76.

18. Ratner V, Starkov A, Matsiukevich D, Polin RA, Ten VS. Mitochondrial dysfunction contributes to alveolar developmental arrest in hyperoxiaexposed mice. Am J Respir Cell Mol Biol 2009;40:511-8.

19. Ratner V, Sosunov SA, Niatsetskaya ZV, Utkina-Sosunova IV, Ten VS. Mechanical ventilation causes pulmonary mitochondrial dysfunction and delayed alveolarization in neonatal mice. Am J Respir Cell Mol Biol 2013;49:943-50.

20. Bizzozero OA, Sanchez P, Tetzloff SU. Effect of ATP depletion on the palmitoylation of myelin proteolipid protein in young and adult rats. J Neurochem 1999;72:2610-6.

21. Schoenfeld R, Wong A, Silva J, et al. Oligodendroglial differentiation induces mitochondrial genes and inhibition of mitochondrial function represses oligodendroglial differentiation. Mitochondrion 2010;10: 143-50.

22. Wang Y, Zhang Y, He J, et al. Hyperforin promotes mitochondrial function and development of oligodendrocytes. J Neurochem 2011;119: $555-68$.

23. Ziabreva I, Campbell G, Rist J, et al. Injury and differentiation following inhibition of mitochondrial respiratory chain complex IV in rat oligodendrocytes. Glia 2010;58:1827-37.

24. Elitt CM, Rosenberg PA. The challenge of understanding cerebral white matter injury in the premature infant. Neuroscience 2014;276:216-38.

25. Gray PH, Burns YR, Mohay HA, O'Callaghan MJ, Tudehope DI. Early postnatal starvation causes lasting brain hypomyelination. Arch Dis Child Fetal Neonatal Ed 1995;73:F128-34.

26. Robain O, Ponsot G. Effects of undernutrition on glial maturation. Brain Res 1978;149:379-97.

27. Brown GK. Congenital brain malformations in mitochondrial disease. J Inherit Metab Dis 2005;28:393-401.

28. Vanopdenbosch L, Dubois B, D’Hooghe MB, Meire F, Carton H. Mitochondrial mutations of Leber's hereditary optic neuropathy: a risk factor for multiple sclerosis. J Neurol 2000;247:535-43.

29. Venditti CP, Harris MC, Huff D, et al. Congenital cardiomyopathy and pulmonary hypertension: another fatal variant of cytochrome-c oxidase deficiency. J Inherit Metab Dis 2004;27:735-9.

30. Chabrol B, Sigaudy S, Paquis V, et al. Stüve-Wiedemann syndrome and defects of the mitochondrial respiratory chain. Am J Med Genet 1997;72:222-6.

31. Thornton C, Hagberg H. Role of mitochondria in apoptotic and necroptotic cell death in the developing brain. Clin Chim Acta 2015;451(Pt A):35-8.

32. Ten VS, Starkov A. Hypoxic-ischemic injury in the developing brain: the role of reactive oxygen species originating in mitochondria. Neurol Res Int 2012;2012:542976.

33. Jones CI 3rd, Han Z, Presley T, et al. Endothelial cell respiration is affected by the oxygen tension during shear exposure: role of mitochondrial peroxynitrite. Am J Physiol Cell Physiol 2008;295:C180-91.

34. Ahmad S, White CW, Chang LY, Schneider BK, Allen CB. Glutamine protects mitochondrial structure and function in oxygen toxicity. Am J Physiol Lung Cell Mol Physiol 2001;280:L779-91.

35. Merker MP, Audi SH, Lindemer BJ, Krenz GS, Bongard RD. Role of mitochondrial electron transport complex I in coenzyme Q1 reduction by intact pulmonary arterial endothelial cells and the effect of hyperoxia. Am J Physiol Lung Cell Mol Physiol 2007;293:L809-19.

36. Das KC. Hyperoxia decreases glycolytic capacity, glycolytic reserve and oxidative phosphorylation in MLE-12 cells and inhibits complex I and II function, but not complex IV in isolated mouse lung mitochondria. PLoS One 2013;8:e73358.

\section{Integrated Mechanism Review}

37. Galam L, Failla A, Soundararajan R, Lockey RF, Kolliputi N. 4-hydroxynonenal regulates mitochondrial function in human small airway epithelial cells. Oncotarget 2015;6:41508-21.

38. Datta A, Kim GA, Taylor JM, et al. Mouse lung development and NOX1 induction during hyperoxia are developmentally regulated and mitochondrial ROS dependent. Am J Physiol Lung Cell Mol Physiol 2015;309: L369-77.

39. Gu S, Chen C, Jiang X, Zhang Z. ROS-mediated endoplasmic reticulum stress and mitochondrial dysfunction underlie apoptosis induced by resveratrol and arsenic trioxide in A549 cells. Chem Biol Interact 2016;245:100-9.

40. He Y, Zou L, Zhou Y, et al. Adiponectin ameliorates the apoptotic effects of paraquat on alveolar type II cells via improvements in mitochondrial function. Mol Med Rep 2016;14:746-52.

41. Niatsetskaya ZV, Sosunov SA, Matsiukevich D, et al. The oxygen free radicals originating from mitochondrial complex I contribute to oxidative brain injury following hypoxia-ischemia in neonatal mice. J Neurosci 2012;32:3235-44.

42. Budinger GR, Tso M, McClintock DS, Dean DA, Sznajder JI, Chandel NS. Hyperoxia-induced apoptosis does not require mitochondrial reactive oxygen species and is regulated by Bcl-2 proteins. J Biol Chem 2002;277:15654-60.

43. Berkelhamer SK, Kim GA, Radder JE, et al. Developmental differences in hyperoxia-induced oxidative stress and cellular responses in the murine lung. Free Radic Biol Med 2013;61:51-60.

44. Ritter J, Schmitz T, Chew LJ, et al. Neonatal hyperoxia exposure disrupts axon-oligodendrocyte integrity in the subcortical white matter. J Neurosci 2013;33:8990-9002.

45. Scheuer T, Brockmöller V, Blanco Knowlton M, et al. Oligodendroglial maldevelopment in the cerebellum after postnatal hyperoxia and its prevention by minocycline. Glia 2015;63:1825-39.

46. Back SA, Craig A, Luo NL, et al. Protective effects of caffeine on chronic hypoxia-induced perinatal white matter injury. Ann Neurol 2006;60: 696-705.

47. Scafidi J, Fagel DM, Ment LR, Vaccarino FM. Modeling premature brain injury and recovery. Int J Dev Neurosci 2009;27:863-71.

48. Scafidi J, Hammond TR, Scafidi S, et al. Intranasal epidermal growth factor treatment rescues neonatal brain injury. Nature 2014;506:230-4.

49. Back SA, Rosenberg PA. Pathophysiology of glia in perinatal white matter injury. Glia 2014;62:1790-815.

50. Durand M, McEvoy C, MacDonald K. Spontaneous desaturations in intubated very low birth weight infants with acute and chronic lung disease. Pediatr Pulmonol 1992;13:136-42.

51. Garg M, Kurzner SI, Bautista DB, Keens TG. Clinically unsuspected hypoxia during sleep and feeding in infants with bronchopulmonary dysplasia. Pediatrics 1988;81:635-42.

52. Cai J, Tuong CM, Zhang Y, et al. Mouse intermittent hypoxia mimicking apnoea of prematurity: effects on myelinogenesis and axonal maturation. J Pathol 2012;226:495-508.

53. Juliano C, Sosunov S, Niatsetskaya Z, et al. Mild intermittent hypoxemia in neonatal mice causes permanent neurofunctional deficit and white matter hypomyelination. Exp Neurol 2015;264:33-42.

54. Douglas RM, Ryu J, Kanaan A, et al. Neuronal death during combined intermittent hypoxia/hypercapnia is due to mitochondrial dysfunction. Am J Physiol Cell Physiol 2010;298:C1594-602.

55. Makarov PR, Wiswedel I, Augustin W, Schild L. Hypoxia/reoxygenationinduced damage to mitochondrial activity is determined by glutathione threshold in astroglia-rich cell cultures. Brain Res 2002;933:91-7.

56. Lee HM, Greeley GH Jr, Englander EW. Sustained hypoxia modulates mitochondrial DNA content in the neonatal rat brain. Free Radic Biol Med 2008;44:807-14.

57. Czerniczyniec A, La Padula P, Bustamante J, Karadayian AG, LoresArnaiz S, Costa LE. Mitochondrial function in rat cerebral cortex and hippocampus after short- and long-term hypobaric hypoxia. Brain Res 2015;1598:66-75.

58. Rone MB, Cui QL, Fang J, et al. Oligodendrogliopathy in Multiple Sclerosis: Low Glycolytic Metabolic Rate Promotes Oligodendrocyte Survival. J Neurosci 2016;36:4698-707. 


\section{Integrated Mechanism Review}

59. Falahati S, Breu M, Waickman AT, et al. Ischemia-induced neuroinflammation is associated with disrupted development of oligodendrocyte progenitors in a model of periventricular leukomalacia. Dev Neurosci 2013;35:182-96.

60. Kapitanović Vidak H, Catela Ivković T, Vidak Z, Kapitanović S. COX-1 and COX-2 polymorphisms in susceptibility to cerebral palsy in very preterm infants. Mol Neurobiol 2016; e-pub ahead of print 19 January 2016.

61. Jin C, Londono I, Mallard C, Lodygensky GA. New means to assess neonatal inflammatory brain injury. J Neuroinflammation 2015;12:180.

62. Wang LY, Tu YF, Lin YC, Huang CC. CXCL5 signaling is a shared pathway of neuroinflammation and blood-brain barrier injury contributing to white matter injury in the immature brain. J Neuroinflammation 2016;13:6.

63. Loeliger M, Inder TE, Shields A, et al. High-frequency oscillatory ventilation is not associated with increased risk of neuropathology compared with positive pressure ventilation: a preterm primate model. Pediatr Res 2009;66:545-50.

64. Loeliger M, Inder T, Cain S, et al. Cerebral outcomes in a preterm baboon model of early versus delayed nasal continuous positive airway pressure. Pediatrics 2006;118:1640-53.

65. Bianco-Batlles MD, Sosunov A, Polin RA, Ten VS. Systemic inflammation following hind-limb ischemia-reperfusion affects brain in neonatal mice. Dev Neurosci 2008;30:367-73.

\section{Ten}

66. Bonora M, De Marchi E, Patergnani S, et al. Tumor necrosis factor- $\alpha$ impairs oligodendroglial differentiation through a mitochondria-dependent process. Cell Death Differ 2014;21:1198-208.

67. Starkov AA, Fiskum G. Regulation of brain mitochondrial $\mathrm{H} 2 \mathrm{O} 2$ production by membrane potential and $\mathrm{NAD}(\mathrm{P}) \mathrm{H}$ redox state. J Neurochem 2003;86:1101-7.

68. Samavati L, Lee I, Mathes I, Lottspeich F, Hüttemann M. Tumor necrosis factor alpha inhibits oxidative phosphorylation through tyrosine phosphorylation at subunit I of cytochrome $\mathrm{c}$ oxidase. J Biol Chem 2008;283:21134-44.

69. Voloboueva LA, Lee SW, Emery JF, Palmer TD, Giffard RG. Mitochondrial protection attenuates inflammation-induced impairment of neurogenesis in vitro and in vivo. J Neurosci 2010;30:12242-51.

70. De Nuccio C, Bernardo A, Cruciani C, De Simone R, Visentin S, Minghetti L. Peroxisome proliferator activated receptor- $\gamma$ agonists protect oligodendrocyte progenitors against tumor necrosis factor-alpha-induced damage: Effects on mitochondrial functions and differentiation. Exp Neurol 2015;271:506-14.

71. Tyler WA, Gangoli N, Gokina P, et al. Activation of the mammalian target of rapamycin (mTOR) is essential for oligodendrocyte differentiation. J Neurosci 2009;29:6367-78. 